\title{
Alfred Ackermann-Teubner-Gedächtnispreis.
}

Der von Herrn Hofrat Dr. Alfred Ackermann-Tenbner in Leipzig im Jahre 1912 bei der Universität Leipzig errichtete "Alfred AckermannTeubner-Gedächtnispreis zur Förderung der mathematisehĕn Wissenschaften" ist in diesem Jahre dureh das Preisgericht Herrn Professor Dr. L. Prandtl in Göttingen für die Arbeit „Über den Luftwiderstand von Kugeln“ (in den Göttinger Nachrichten von 1914) zuerkannt worden.

Nachträgliche Bemerkung über die Erweiterung des Definitionsbereichs einer stetigen Funktion.

\section{Von}

L. E. J. Brouwer in Amsterdam.

Die Erweiterung des Definitionsbereichs einer auf einer ahgeschlossenen Punktmenge definierten stetigen Funktion, welche, wie ich S. 209-211 dieses Annalenbandes hervorgehoben habe, durch Spezialisierang einer Math. Ann. 71, S. 309-311 von mir definierten Konstruktion erhalten wird, ist seitdem ebenfalls, nicht nur, wie angegeben, von De la Vallée Poussin und Bohr, sondern auch von Tietze im Journ. f. Math. 145, S. 10-11 erzielt worden, und zwar steht Tietzes Methode der meinigen sehr nahe und besitzt den gleichen elementaren Charakter: es werden nur, statt Simplexe, Kuben gebraucht.

\section{Berichtigung}

zu dem Aufsatze von L. E. J. Brouwer: „Über eineindeutige, stetige Transformationen von Flächen in sich." Math. Ann. 69, S. 176-180.

S.179,Z.19 zu streichen: miteinander zusammenhängenden

Z. 20-21 zu streichen: mit einem Rande zusammenhängenden

Z. 30-31 zu streichen: der Cartesischen Ebene in sich oder des Kreiszylinders in sich

Z. 32 statt: in solche Gebiete zerlegen za lesen: durch solche Gebiete erschöpfen. 
Wie alle wissenschaftichen Zeitschriften, die in beschränkter Auflage ersckeinen und ihre Abbonnenten zum großen Teil im Ausland haben, so sind auch die Annalen durch den Ausfall dieser, wie andrerseits durch die sich ins Ungemessene steigernden Herstellungskosten in ihrer Weiterführung schwer betroffen. Der Verlag will auch weiterhin die so beträchtlich erhöhten Opfer, die ihm diese auferlegt, nicht scheuen, um.den Weiterbestand der für die Pflege der mathematischen Wissenschaft und ihrer internationalen Beziehungen so wichtigen Zeitschrift, die mit dem nächsten in August d. F. erscheinenden Héft ihren achtzigsten Fahrgang beginnt, zu ermöglichen. Die Redaktion und der Verlag bitten darum den schtvierigen Verhältnissen gegenüber, die auf das regelmäßige Erscheinen und den Umfang der Hefte nicht ohne Einfluß bleiben konnten, gütige Nachsicht zu üben und sie in ihrem Bestreben, den Annalen über die schwierigen Zeiten hinwegzuhelfen, zu unterstützen. Dann hoffen sie, unter gü̈nstigeren Bedingungen sie zu newer Entroicklung zu bringen und damit auch dem Ansehen der deutschen wissenschaftlichen Arbeit im Ausland förderlich zu sein.

\section{Redaktion und Verlag.}

